\title{
HCN SPECTROSCOPY OF COMET 73P/SCHWASSMANN-WACHMANN 3. A STUDY OF GAS EVOLUTION AND ITS LINK TO CN
}

\author{
L. Paganini ${ }^{1,6}$, G. L. Villanueva ${ }^{2}$, L. M. Lara ${ }^{3}$, Z. Y. Lin $^{3}$, M. Küppers ${ }^{4}$, P. Hartogh $^{1}$, And A. Faure $^{5}$ \\ ${ }^{1}$ Max-Planck-Institut für Sonnensystemforschung, Max-Planck-Strasse 2, D-37191 Katlenburg-Lindau, Germany; lucas.paganini@ nasa.gov \\ ${ }^{2}$ Solar System Exploration Division, NASA Goddard Space Flight Center, Greenbelt, MD 20771, USA \\ ${ }^{3}$ Instituto de Astrofísica de Andalucía, CSIC, Camino Bajo de Huétor 50, 18008 Granada, Spain \\ ${ }^{4}$ European Space Astronomy Center (ESAC), P.O. Box 78, 28691 Villanueva de la Cañada, Madrid, Spain \\ ${ }^{5}$ Laboratoire d'Astrophysique de Grenoble (LAOG), Université Joseph-Fourier, UMR 5571 CNRS, BP 53, 38041 Grenoble Cedex 09, France \\ Received 2010 January 20; accepted 2010 April 14; published 2010 May 7
}

\begin{abstract}
In 2006 May, comet 73P/Schwassmann-Wachmann 3 experienced large outburst activity allowing us to study the gas production rate of fresh material released from the nucleus. We observed the comet in a coordinated campaign using millimeter and optical facilities at heliocentric distances between 0.966 and 1.033 AU. During this time, we had the opportunity to follow the post-outburst evolution of fragment $\mathrm{B}$, which evidenced larger production rates in comparison to fragment $\mathrm{C}$, the latter showing a rather stable gas production rate $\left(Q_{\mathrm{HCN}} \sim 2 \times 10^{25}\right.$ molecules $\mathrm{s}^{-1}$ ). In addition to the investigation of the gas evolution, we studied the possible role of $\mathrm{HCN}$ and dust as progenitors for the $\mathrm{CN}$ radical. From our joint observations on May 12, we observed a high correlation of $\mathrm{CN}$ with $\mathrm{HCN}$ and low correlation with the continuum emission (grains). Herewith, our study supports the view of $\mathrm{HCN}$ as a major source of $\mathrm{CN}$, although the presence of other sources for cyanide cannot be fully ruled out.
\end{abstract}

Key words: astrochemistry - comets: general - comets: individual (73P/Schwassmann-Wachmann 3) - molecular processes - radiative transfer

Online-only material: color figures

\section{INTRODUCTION}

Cometary nuclei are considered primordial leftovers from the early time of our solar system, so the composition of their native ices represents comprehensive evidence of different thermochemical and physical conditions that occurred 4.6 billion years ago, the time when our planetary system was formed. Comets reside in two dynamical reservoirs: the Oort Cloud (source of nearly isotropic comets: long period and Halley type) and the Kuiper Belt (source of ecliptic comets: Jupiter-family comets, Encke type, and Centaur type). Individual comets from these reservoirs can be perturbed toward the inner solar system by various gravitational effects. As a comet approaches the Sun, volatile ices sublimate, and the ejected molecules can be spectroscopically detected, for instance, by observing their rotational or rovibrational transitions at submillimeter wavelengths.

The Jupiter-family comet 73P/Schwassmann-Wachmann 3 (hereafter 73P) constitutes one of the most relevant objects for cometary research. Discovered by Friedrich Carl Arnold Schwassmann and Arno Arthur Wachmann at the Hamburg Observatory in 1930, 73P is currently known for a large occurrence of outburst and defragmentation events. In particular, 73P broke up into several pieces during its perihelion passage in 1995. As a consequence, four fragments were observed and named alphabetically (Böhnhardt et al. 1995). The fourth one, however, was not seen elsewhere and thus might have been an ephemeral debris. In 2006, its close approach to Earth $(\Delta=$ $0.07 \mathrm{AU}$ ) prompted astronomical observers to organize multiple observation campaigns using ground- and space-based facilities (e.g., Villanueva et al. 2006; Dello Russo et al. 2007; Fuse et al. 2007; Jones et al. 2008; Reach et al. 2009). During this time,

\footnotetext{
6 Present address: Solar System Exploration Division, NASA Goddard Space Flight Center, Mailstop 693.0, Greenbelt, MD 20771, USA.
}

73P again experienced outbursts and nucleus defragmentation producing over three dozen pieces, which were observed before its perihelion passage. Clearly, the importance of these outburst episodes relies on the possibility of analyzing the fresh material in the coma which might thus enhance the study of intrinsic properties of cometary nuclei.

In 2006 May, we observed comet 73P/SchwassmannWachmann 3 using the $10 \mathrm{~m}$ Submillimeter Telescope. During this time, we had the unique opportunity to follow the post-outburst evolution of fragment B. Indeed, the advantage of radio spectroscopy relies on its capability to characterize the cometary coma and to determine, for instance, its chemical taxonomy, expansion velocities, kinetic temperatures, and production rates. Supporting observations with the $1 \mathrm{~m}$ telescope at the Lulin Observatory provided complementary information at optical wavelengths. The observed components were mainly fragments $\mathrm{B}$ and $\mathrm{C}$ focusing on molecular species, e.g., $\mathrm{HCN}, \mathrm{HNC}, \mathrm{CH}_{3} \mathrm{OH}, \mathrm{CO}, \mathrm{H}_{2} \mathrm{CO}, \mathrm{CS}, \mathrm{CN}$, and the isotopic ratio ${ }^{13} \mathrm{~N} /{ }^{14} \mathrm{~N}$ in $\mathrm{HCN}$. A complete report of the comet's chemical taxonomy will be presented elsewhere (G. L. Villanueva et al. 2010, in preparation). A previous study by Drahus et al. (2010) focused on the analysis of the rotation period of fragment $\mathrm{C}$ based on the analysis of short-term variabilities in HCN production rates. Among several plausible solutions for periodicity, a rotation period of $3.2 \pm 0.2 \mathrm{hr}$ was suggested to be the most likely.

In this publication, we seek to tackle two topics. First, we present the gas evolution of fragments B and C at heliocentric distances, $r_{h}$, from UT 2006 May 9 to May 22. This is performed by retrieving the gas production rates, kinetic temperature, and expansion velocity, using a recently developed analysis package called CERT, Cometary Excitation and Radiative Transfer (L. Paganini 2010, in preparation). CERT is a numerical code suitable for the simulation of emission lines at millimeter and 
submillimeter wavelengths in cometary comae, the latter including local thermodynamic equilibrium (LTE) and non-LTE conditions, i.e., collisions and radiative processes. The radiation process considers excitation of the $\mathrm{HCN}$ population distribution by absorption of solar photons via the $v_{1}, v_{2}, 2 v_{2}$, and $v_{3}$ vibration bands. Second, we investigate the linkage between $\mathrm{HCN}$ and $\mathrm{CN}$ using our joint observations at millimeter wavelengths and $\mathrm{CN}$ imaging from the optical. Here, we analyze the consistency in production rates of $\mathrm{HCN}$ and $\mathrm{CN}$, and further discuss the jet morphology in their radial distribution.

This paper is organized as follows. Section 2 details the instrumentation and observations. In Section 3, we give a short description of the excitation and radiative transfer code used during data analysis. The results of these observations and analysis, presented in Section 4, provide information regarding the gas production rates of HCN (Section 4.1) and CN (Section 4.2), and a comparison of jet morphologies between $\mathrm{HCN}$ mapping at millimeter wavelengths and $\mathrm{CN}$ images at optical wavelengths on 2006 May 12 (Section 4.3). Next, possible parent species for the $\mathrm{CN}$ radical are discussed in Section 5. Finally, Section 6 presents the conclusions for this work.

\section{OBSERVATIONS}

\subsection{Submillimeter Wavelengths}

Observations were performed with the Heinrich Hertz Submillimeter Telescope (HHSMT) between UT 2006 May 9 and 22. The telescope is located on Mt. Graham in southeast Arizona at an altitude of $3200 \mathrm{~m}$ (longitude W 109 53'26", latitude N $\left.32^{\circ} 42^{\prime} 05^{\prime \prime}\right)$. The HHSMT consists of a Cassegrain system with a paraboloidal main reflector and a hyperboloidal secondary reflector. The antenna has a diameter of $10 \mathrm{~m}$, the absolute pointing accuracy is about $2^{\prime \prime}$, with a tracking accuracy of better than $1^{\prime \prime}$ (Baars et al. 1999); however, we have observed pointing errors of up to $\sim 8^{\prime \prime}$. The observations were performed using the dual polarization, single sideband $1.3 \mathrm{~mm}$ JT (130-300 GHz) and the dual-channel, double sideband MPIfR SIS-345 receivers. The sensitivity of these instruments yielded mean system temperature $\left(T_{\text {sys }}\right)$ of $\sim 500 \mathrm{~K}$ during the observation campaign. The telescope is equipped with several back ends: (1) two chirp transform spectrometers (CTS) of (a) $218 \mathrm{MHz}$ bandwidth (BW) $46.6 \mathrm{kHz}$ spectral resolution $(\Delta f)$ and (b) $\mathrm{BW}=400 \mathrm{MHz} \Delta f$ $=100 \mathrm{kHz}$; (2) a set of three acousto-optical spectrometers (AOSs), (a) $\mathrm{AOS}_{A}: \Delta f=934 \mathrm{kHz}, \mathrm{BW}=1 \mathrm{GHz}$, (b) $\mathrm{AOS}_{B}: \Delta f$ $=913 \mathrm{kHz}, \mathrm{BW}=1 \mathrm{GHz},(\mathrm{c}) \mathrm{AOS}_{C}: \Delta f=370 \mathrm{kHz}, \mathrm{BW}=$ $250 \mathrm{MHz}$; and (3) several filter banks.

We report astronomical observations of $\mathrm{HCN}$ species, transitions $J=3-2(v=265886.1800 \mathrm{MHz})$ and $J=4-3(v$ $=354505.4759 \mathrm{MHz}$; Pickett et al. 1998). The half-power beamwidth (HPBW) for these transitions corresponds to $29^{\prime \prime}$ and $22^{\prime \prime}$, respectively. In this study, we use observations by the $218 \mathrm{MHz}$ BW chirp transform spectrometer, which provides a velocity resolution of $\sim 0.05 \mathrm{~km} \mathrm{~s}^{-1}$ at $v=250 \mathrm{GHz}$. The spectral data are automatically calibrated by the telescope software into antenna temperature scale $\left(T_{A}^{*}\right)$ using the chopperwheel method (Ulich \& Haas 1976). The observing mode for cometary observations was position switching (PS) which consists on pointing the telescope to the source (on-position) for an integration time of $15 \mathrm{~s}$, and afterward the telescope is directed to the background (off-position) for a same integration time. A complete scan was finished after 5 minutes. After each $1 \mathrm{hr}$ observing interval, observations of appropriate astronomical standard stars were acquired for pointing-accuracy determina- tion and flux calibration. Afterward, the scanning procedure was repeated; i.e., the comet was observed again for $1 \mathrm{hr}$. Telescope tracking of the comet was computed using daily updated orbital elements from the JPL Horizons Ephemeris System ${ }^{7}$ (Table 1 shows the log of the observations). Main-beam efficiencies were attained from continuum observations of Venus, Mars, Jupiter, and Saturn, and line observations of G34.3, DR21, CRL2688, and W51d.

\subsection{Optical Wavelengths}

At optical wavelengths, our observations were obtained with the $1 \mathrm{~m}$ telescope at the Lulin Observatory, National Central University, Taiwan equipped with a PI1300B $1340 \times 1300$ CCD camera with an effective pixel scale of 0 .'515 pixel $^{-1}$ (Kinoshita et al. 2005). These observations were performed with the ESA Rosetta comet filter set for $\mathrm{CN}$ at $387 / 6 \mathrm{~nm}$ and the blue continuum at $445 / 4 \mathrm{~nm}$ (Table 2). The telescope tracking speed was set to the proper motion of the comet on the sky. The image data reduction has followed standard procedures which began with bias and dark current subtraction, and flat-field correction for all frames. The night sky contribution was subtracted from the comet images themselves at positions where no clear comet contribution was detected. This can easily be done for the images acquired with the BC filter as the dust coma does not fill the field of view (FOV). However, for the gas images, we estimated the sky contribution from the SW edge of the frame where no clear gas emission can be detected. The amount of sky background measured only represents $2 \%$ of the comet emission. We have used the spectrophotometric standard star BD+28D4211 for calibration into physical units. After every individual comet image is flux-calibrated, a final resulting comet image from the $\mathrm{CN}$ and the $\mathrm{BC}$ filters is obtained by median combining the complete CN and BC set of frames (see Lin et al. 2007 and 2009 for further details).

\section{HCN EXCITATION AND RADIATIVE TRANSFER MODEL}

A convenient method to determine the chemical taxonomy of comets - a classification based on the nature of ices and dust that comprise the nucleus-is to observe the parent volatiles (and refractory grains) shortly after their release from the cometary nucleus. As a comet approaches the Sun, the bulk composition of cometary nuclei can be analyzed by radio spectroscopy. The analysis of cometary comae by this method allows quantification of parent molecules with high spectral resolution.

Hydrogen cyanide is one of the best-studied molecules at radio wavelengths. Since the HCN molecule has a large dipole moment, and thus a short rotational lifetime, its LTE region is relatively small in the cometary coma. A proper extraction of their absolute production rates and mixing ratios (relative populations) from the measured line intensities requires a comprehensive investigation of the excitation processes and radiative transfer in the coma. As described by Crovisier (1984) and Weaver \& Mumma (1984), the main excitation mechanisms within the cometary coma are collisions and radiative processes. In the first case, excitation involves collisions of the neutral gas with water vapor and electrons, controlling rotational populations in the inner coma, up to a cometocentric radius of $10^{2}-10^{4} \mathrm{~km}$ depending on cometary activity (Xie \& Mumma 1992). Radiative processes are important for excitation throughout the coma, but

\footnotetext{
http://ssd.jpl.nasa.gov/?horizons
} 
Table 1

Log of Observations at Millimeter and Optical Wavelengths

\begin{tabular}{|c|c|c|c|c|c|c|c|}
\hline & $\begin{array}{c}\text { Date } \\
\text { (2006 UT) }\end{array}$ & $\begin{array}{c}r_{h} \\
(\mathrm{AU}) \\
\end{array}$ & $\begin{array}{c}\Delta \\
(\mathrm{AU})\end{array}$ & $\begin{array}{l}\text { P.A. }^{a} \\
\text { (deg) }\end{array}$ & $\begin{array}{c}\alpha^{\mathrm{b}} \\
(\mathrm{deg})\end{array}$ & $\begin{array}{l}\mathrm{HCN} \\
(\mathrm{UT}) \\
\end{array}$ & $\begin{array}{c}\mathrm{CN} \\
(\mathrm{UT}) \\
\end{array}$ \\
\hline \multirow[t]{7}{*}{ Fragment B } & May 9 & 1.033 & 0.078 & 229.9 & 70.5 & $10: 54-12: 18$ & $\ldots$ \\
\hline & May 10 & 1.027 & 0.074 & 235.1 & 74.8 & $12: 13-13: 31$ & $\ldots$ \\
\hline & May 11 & 1.020 & 0.070 & 240.4 & 79.5 & $15: 19-15: 36$ & $\ldots$ \\
\hline & May 12 & 1.015 & 0.068 & 244.8 & 83.7 & $07: 53-17: 02$ & $18: 17-19: 28$ \\
\hline & May 17 & 0.990 & 0.072 & 256.1 & 105.1 & $09: 45-19: 20$ & $\ldots$ \\
\hline & May 20 & 0.976 & 0.086 & 253.1 & 112.1 & $18: 47-19: 28$ & $\ldots$ \\
\hline & May 21 & 0.973 & 0.091 & 251.9 & 113.0 & $10: 35-19: 50$ & $\ldots$ \\
\hline \multirow[t]{7}{*}{ Fragment C } & May 9 & 1.027 & 0.083 & 242.1 & 75.3 & $06: 32-15: 42$ & $\ldots$ \\
\hline & May 10 & 1.021 & 0.080 & 245.4 & 79.4 & $07: 33-15: 28$ & $\ldots$ \\
\hline & May 11 & 1.015 & 0.079 & 248.8 & 84.2 & $15: 55-17: 04$ & $\ldots$ \\
\hline & May 12 & 1.010 & 0.079 & 250.8 & 87.8 & $09: 03-17: 23$ & $19: 46-20: 19$ \\
\hline & May 17 & 0.986 & 0.090 & 253.8 & 104.1 & $11: 31-16: 55$ & $\ldots$ \\
\hline & May 20 & 0.973 & 0.105 & 250.8 & 108.7 & $10: 54-18: 39$ & $\ldots$ \\
\hline & May 22 & 0.966 & 0.117 & 248.6 & 110.0 & $10: 59-12: 15$ & $\ldots$ \\
\hline
\end{tabular}

Notes.

a Solar position angle.

b Solar phase angle.

they determine rotational populations only in the outer coma (i.e., beyond a cometocentric radius of $10^{4} \mathrm{~km}$ ), where there exists a balance (fluorescence equilibrium) between rovibrational levels absorbing solar radiation and the subsequent spontaneous decay into excited rotational levels in the ground vibrational state (which also experience radiative cooling).

Due to a larger FOV, numerical simulations of millimeter and submillimeter observations need to account for all excitation processes and non-LTE conditions within the coma. We have analyzed 73P data using a recently developed analysis package called CERT. CERT is a numerical code suitable for the simulation of emission lines at millimeter and submillimeter wavelengths in cometary comae, the latter including LTE and non-LTE conditions. In addition, CERT considers the actual heliocentric and geocentric distances of the comet and, if available, possible telescope offsets. Basically, the following points describe the different mechanisms composing this code.

First, a molecular excitation model considers spherically symmetric outflow to describe the spatial density distribution of parent species ejected from the nucleus (including their photodissociation rate). The radial density distribution is calculated for a large number of cells within the coma up to a cometocentric radius of $10^{6} \mathrm{~km}$ extending equally in logarithmic space. The excitation characteristics within each cell are considered constant, and the molecular density is defined as

$$
n_{\mathrm{HCN}}(r)=\frac{Q_{\mathrm{HCN}}}{4 \pi r^{2} v_{\exp }} e^{\frac{-\left(r-r_{0}\right)}{\lambda_{p}}},
$$

where $Q_{\mathrm{HCN}}$ is the production rate, $r$ is cometocentric radius, $v_{\text {exp }}$ is the gas expansion velocity, $r_{0}$ is the nucleus radius (assumed to be $500 \mathrm{~m}$ for fragments $\mathrm{B}$ and $\mathrm{C}$ ), and $\lambda_{p}$ is the photodissociation scale length. $\lambda_{p}$ is equal to $v_{\exp } / \beta_{\mathrm{HCN}}$, where $\beta_{\mathrm{HCN}}=\beta_{0} / r_{h}^{2}$, and the dissociation rate at $1 \mathrm{AU}$ heliocentric distance, $\beta_{0}$, is $1.6 \times 10^{-5} \mathrm{~s}^{-1}$ at solar minimum (Biver et al. 1999). Even though CERT handles temperature and velocity profiles (e.g., Crovisier 1984; Combi et al. 1999), we use constant values to describe the expansion velocity and kinetic temperature in the cometary coma.

A radiative transfer code is applied based on the existing accelerated Monte Carlo approach for star formation models
Table 2

Characteristics of the Observed Species

\begin{tabular}{lcc}
\hline \hline Species & Transition & Wavelength \\
\hline $\mathrm{HCN}$ & $3-2$ & $1.13 \mathrm{~mm}$ \\
& $4-3$ & $0.85 \mathrm{~mm}$ \\
\hline $\mathrm{CN}$ & $\mathrm{B}^{2} \Sigma^{+}-\mathrm{X}^{2} \Sigma^{+}(0,0)$ & $387 / 6 \mathrm{~nm}$ \\
Dust & $\cdots$ & $445 / 4 \mathrm{~nm}$ \\
\hline
\end{tabular}

by Hogerheijde \& van der Tak (2000). This code has been adapted for cometary applications, similarly as by Bensch \& Bergin (2004). Nevertheless, CERT uses a novel approach which greatly improves the overall execution speed of Monte Carlo methods by parallel processing using the Message Passing Interface (MPI).

The radiative transfer equation describes the emission, absorption, and motion of photons along a line in the direction of propagation, and is defined as

$$
\frac{d I_{v}}{d \tau_{v}}=S_{\nu}-I_{\nu},
$$

where $S_{v}$ is the source function, $I_{v}$ represents the specific intensity of electromagnetic radiation, and $d \tau_{\nu}=\alpha_{\nu} d s$ the optical depth. In a two-level system, the molecular line transfer is determined by transition rates between two molecular rotational levels, $i$ (upper) and $j$ (lower), characterized by their specific population, $n_{i}$ and $n_{j}$. The source function is thus given by $S_{i j}(v)=j_{i j}(v) / \alpha_{i j}(v)$, where $j_{i j}(v)$ and $\alpha_{i j}(v)$ stand for emission and absorption coefficients, given by

$$
\begin{gathered}
j_{i j}(v)=\frac{h v_{o}}{4 \pi} n_{i} A_{i j} \phi(v), \\
\alpha_{i j}(v)=\frac{h v_{o}}{4 \pi}\left(n_{j} B_{j i}-n_{i} B_{i j}\right) \phi(v) .
\end{gathered}
$$

The Einstein coefficients for spontaneous emission and absorption, $A_{i j}$ and $B_{j i}$, and stimulated emission, $B_{i j}$, are obtained from a molecular line atlas. The function $\phi(v)$ describes the line profile function. 
The relative level populations are obtained from the statistical equilibrium equation,

$$
\begin{aligned}
n_{i} & {\left[\sum_{j<i} A_{i j}+\sum_{j \neq i}\left(B_{i j} J_{v}+C_{i j}+G_{i j}\right)\right] } \\
& =\sum_{j>i} n_{j} A_{j i}+\sum_{j \neq i} n_{j}\left(B_{j i} J_{v}+C_{j i}+G_{j i}\right),
\end{aligned}
$$

which is solved by iteration, using matrix inversion and LU decomposition, until the solution has arrived to a satisfactory value (Hogerheijde \& van der Tak 2000). $J_{v}$ is the mean intensity, which is the integrated specific intensity from all solid angles, $d \Omega$. The collisional rate is $C_{i j}=n_{\mathrm{cp}} k_{i j}$ with $k_{i j}$ being the collision rate coefficients and $n_{\mathrm{cp}}$ the density of the collision partners. The contribution of radiative processes is determined by "effective" pumping rates $G_{i j}$ (Bensch $\&$ Bergin 2004), using fluorescence models (in the CERT package).

The excitation of molecular rotational levels is modeled for collisions of hydrogen cyanide with water and electrons, using theoretical cross sections $(\sigma)$. Since there are no detailed cross sections for water and hydrogen cyanide, i.e., relative to their rotational level properties, we have followed the standardized method of using a constant $\sigma_{\mathrm{H}_{2} \mathrm{O}-\mathrm{HCN}}$ equal to $1 \times 10^{-14} \mathrm{~cm}^{2}$ (Biver et al. 1999). Furthermore, the contribution of collisional excitation by thermal electron impact has been calculated using the molecular $R$-matrix method combined with the adiabaticnuclei-rotation (ANR) approximation. We consider $e$-HCN rotation rates for transitions among all levels up to $J=8$, and the fit of Faure et al. (2007) was employed up to $T=10,000 \mathrm{~K}$. The electron density and temperature profiles are derived from measurements in the coma of $1 \mathrm{P} /$ Halley following the study by Biver (1997); we use a scaling factor $x_{n e}=0.3$.

The contribution of radiative processes to the line intensity is determined by detailed emission rates using a fluorescence model, the so-called $g$-factors. The calculation of $g$-factors is based on ab initio parameters, such as Einstein A-coefficients, statistical weights, and line strengths. The detailed pumping rate, due to the solar infrared radiation at a temperature $T_{b b}=$ $5770 \mathrm{~K}$ and having solid angle $\Omega_{b b}$, is

$$
g_{j i}=\frac{\Omega_{b b}}{4 \pi} P_{j} \frac{\omega_{i}}{\omega_{j}} A_{i j}\left(e^{\frac{h c \sigma_{j i}}{k T_{b b}}}-1\right)^{-1},
$$

where $P_{j}$ is the rotational population of the lower level $j, \omega_{i}$ and $\omega_{j}$ are the statistical weights of the upper and lower levels, $h$ is Planck's constant, $k$ is Boltzmann's constant, $c$ is the speed of light, and $\sigma_{j i}$ is the frequency (see Crovisier \& Encrenaz 1983 for further details).

CERT only considers cold (or fundamental) bands and accesses the HITRAN 2008 Molecular Line Atlas (Rothman et al. 2009) to obtain the required molecular parameters (which are attuned to the corresponding rotational temperature). Following Bockelée-Morvan et al. (1984), the radiation process considers excitation of the HCN population distribution by absorption of solar photons via the $v_{1}, v_{2}, 2 v_{2}$, and $v_{3}$ vibration bands (accounting for 40 rotational levels), and the subsequent spontaneous decay into the excited rotational levels in the ground vibrational state. The l-type splitting of the $2 v_{2}$ rotational levels was considered; however, hyperfine structures have been ignored. Excitation via $v_{3}$ has been observed to be negligible. In addition, the outer coma is considered optically thin, so here any opacity effects have been neglected.

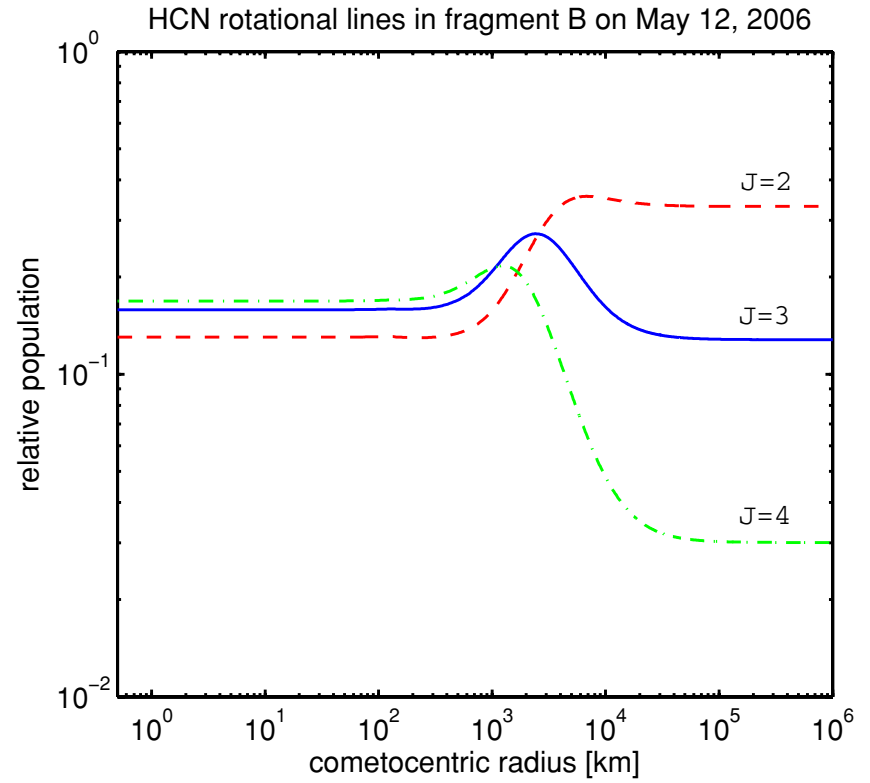

Figure 1. Level population of HCN corresponding to fragment B on 2006 May 12. This calculation considers: (1) a cometary coma extending from $500 \mathrm{~m}$ up to $10^{6} \mathrm{~km}$, (2) a constant kinetic temperature of $90 \mathrm{~K}$, (3) interaction with water vapor and electrons, and (4) contribution of radiative processes of 40 rotational levels within vibrational bands $v_{1}, v_{2}, 2 v_{2}$, and $v_{3}$ into the ground vibrational state.

(A color version of this figure is available in the online journal.)

Following Bensch \& Bergin (2004), the effective pumping rate is computed from the detailed contribution of cascading rotational levels $\left(g_{i j}\right)$, within the considered vibrational bands (i.e., $v_{1}, v_{2}, 2 v_{2}$, and $v_{3}$ ), into each excited rotational level in the ground vibration state:

$$
G_{i j}=\sum_{J^{\prime}, v^{\prime}} g_{i j}\left(J^{\prime}, v^{\prime}\right)
$$

Figure 1 displays an example of the rotational population distribution corresponding to 73P-B on 2006 May 12 (cf. Figure 1 in Bockelée-Morvan et al. 2004). These level populations have been computed using a constant temperature along the coma equal to $90 \mathrm{~K}$. We can observe an evolution from thermal to fluorescence equilibrium in the cometary atmosphere. The SMT FOV for the $J=3-2$ rotational transition equals $1662 \mathrm{~km}$ and $1161 \mathrm{~km}$ for $J=4-3$ (at their proper frequencies and $\Delta=$ $0.079 \mathrm{AU}$ ). These FOVs correspond to cometocentric radii of 831 and $581 \mathrm{~km}$, respectively. Thus, we note that both FOV configurations are mostly sensitive to the inner coma where thermal equilibrium predominates. A temperature variation, however, is estimated to occur according to kinetic models (Crovisier 1984). This variation accounts for cooling due to gas adiabatic expansion close to the coma and a temperature increase due to solar heating at larger distances.

\section{RESULTS}

\subsection{HCN Production Rates: Evolution of Cometary Outgassing}

The measured HCN spectra were reduced using CLASS from the GILDAS software package. ${ }^{8}$ The synthetic HCN line emission is calculated after ray tracing using SKY (Hogerheijde

\footnotetext{
8 http://www.iram.fr/IRAMFR/GILDAS
} 
Table 3

73P-B Production Rates

\begin{tabular}{|c|c|c|c|c|c|c|c|}
\hline Molecule & $\begin{array}{l}\text { Date } \\
\text { (UT) }\end{array}$ & $\begin{array}{c}r_{h} \\
(\mathrm{AU})\end{array}$ & $\begin{array}{c}\Delta \\
(\mathrm{AU})\end{array}$ & $\begin{array}{c}\int T_{b} d v^{\mathrm{a}} \\
\left(\mathrm{K} \mathrm{km} \mathrm{s}^{-1}\right)\end{array}$ & $\begin{array}{c}Q_{\mathrm{HCN}} \\
\left(10^{25} \mathrm{~s}^{-1}\right)\end{array}$ & $\begin{array}{c}Q_{\mathrm{CN}} \\
\left(10^{25} \mathrm{~s}^{-1}\right)\end{array}$ & $\begin{array}{c}\frac{Q_{\mathrm{HCN}} \mathrm{b}}{Q_{\mathrm{H}_{2} \mathrm{O}}} \\
(\%) \\
\end{array}$ \\
\hline \multirow[t]{6}{*}{$\mathrm{HCN}(3-2)$} & May 9 & 1.033 & 0.078 & $0.96 \pm 0.04$ & & $\ldots$ & $\ldots$ \\
\hline & May 11 & 1.020 & 0.070 & $2.40 \pm 0.09$ & $3.23 \pm 0.25$ & $\ldots$ & 0.17 \\
\hline & May 12 & 1.015 & 0.068 & $2.33 \pm 0.06$ & $3.04 \pm 0.16$ & $6.42 \pm 0.74$ & 0.16 \\
\hline & May 17 & 0.990 & 0.072 & $1.62 \pm 0.10$ & $2.21 \pm 0.30$ & $\ldots$ & 0.20 \\
\hline & May 20 & 0.976 & 0.086 & $0.90 \pm 0.14$ & $1.30 \pm 0.44$ & $\ldots$ & 0.20 \\
\hline & May 21 & 0.973 & 0.091 & $0.65 \pm 0.05$ & $0.97 \pm 0.16$ & $\ldots$ & 0.20 \\
\hline $\mathrm{HCN}(4-3)$ & May 10 & 1.027 & 0.074 & $7.11 \pm 0.08$ & $5.93 \pm 0.15$ & $\ldots$ & 0.31 \\
\hline
\end{tabular}

Notes.

a Line intensities are calculated using a velocity interval equal to $\pm 1 \mathrm{~km} \mathrm{~s}^{-1}$.

$\mathrm{b}$ The water production rate is $1.9 \times 10^{28} \mathrm{~s}^{-1}$ on May 10-12 (from Kobayashi et al. 2007). Because of the large variability observed in fragment B at infrared wavelengths, we use a constant $Q_{\mathrm{HCN}} / Q_{\mathrm{H}_{2} \mathrm{O}}=0.2 \%$ after May 12 .

Table 4

73P-C Production Rates

\begin{tabular}{|c|c|c|c|c|c|c|c|}
\hline Molecule & $\begin{array}{l}\text { Date } \\
\text { (UT) }\end{array}$ & $\begin{array}{c}r_{h} \\
(\mathrm{AU}) \\
\end{array}$ & $\begin{array}{c}\Delta \\
(\mathrm{AU})\end{array}$ & $\begin{array}{c}\int T_{b} d v^{\mathrm{a}} \\
\left(\mathrm{K} \mathrm{km} \mathrm{s}^{-1}\right)\end{array}$ & $\begin{array}{c}Q_{\mathrm{HCN}} \\
\left(10^{25} \mathrm{~s}^{-1}\right) \\
\end{array}$ & $\begin{array}{c}Q_{\mathrm{CN}} \\
\left(10^{25} \mathrm{~s}^{-1}\right)\end{array}$ & $\begin{array}{c}\frac{Q_{\mathrm{HCN}} \mathrm{b}}{Q_{\mathrm{H}_{2} \mathrm{O}}} \\
(\%) \\
\end{array}$ \\
\hline \multirow[t]{6}{*}{$\mathrm{HCN}(3-2)$} & May 9 & 1.027 & 0.083 & $1.30 \pm 0.02$ & $2.20 \pm 0.07$ & & 0.19 \\
\hline & May 11 & 1.015 & 0.079 & $1.23 \pm 0.06$ & $1.97 \pm 0.22$ & $\ldots$ & 0.17 \\
\hline & May 12 & 1.010 & 0.079 & $1.13 \pm 0.04$ & $1.83 \pm 0.13$ & $2.06 \pm 0.34$ & 0.16 \\
\hline & May 17 & 0.986 & 0.090 & $1.05 \pm 0.06$ & $1.90 \pm 0.22$ & $\ldots$ & 0.17 \\
\hline & May 20 & 0.973 & 0.105 & $0.91 \pm 0.04$ & $1.86 \pm 0.18$ & $\ldots$ & 0.16 \\
\hline & May 22 & 0.966 & 0.117 & $1.21 \pm 0.10$ & $2.74 \pm 0.46$ & $\ldots$ & 0.24 \\
\hline $\mathrm{HCN}(4-3)$ & May 10 & 1.021 & 0.080 & $2.06 \pm 0.05$ & $2.09 \pm 0.11$ & $\ldots$ & 0.18 \\
\hline
\end{tabular}

Notes.

${ }^{\text {a }}$ Line intensities are calculated using a velocity interval equal to $\pm 1 \mathrm{~km} \mathrm{~s}^{-1}$.

$\mathrm{b}$ The water production rate is $1.14 \times 10^{28} \mathrm{~s}^{-1}$ (from Dello Russo et al. 2007), assumed to be constant over the whole observation campaign.

\& van der Tak 2000) and later analyzed with the MIRIAD package (Sault et al. 1995). The resulting HCN brightness distribution is convolved with a beam of $29^{\prime \prime}$ for the $J=$ 3-2 and $22^{\prime \prime}$ for the $J=4-3$, which are the representative HPBWs of the Submillimeter Telescope at their corresponding frequencies. Next, a power-law fit obtains an equation for simulated production rates as a function of synthetic lineintegrated intensities. Afterward, the $\mathrm{HCN}$ production rate is retrieved from this derived equation by introducing the observed line-integrated intensities.

Production rates have been estimated from heliocentric distances between 0.966 and 1.033 AU before perihelion. An expansion velocity, $v_{\text {exp }}$, of $0.53 \mathrm{~km} \mathrm{~s}^{-1}$ and a kinetic temperature of $90 \mathrm{~K}$ were retrieved for $73 \mathrm{P}-\mathrm{B}$. Accordingly, determination of these values for fragment $\mathrm{C}$ yielded a $v_{\exp }$ of $0.63 \mathrm{~km} \mathrm{~s}^{-1}$ and $T_{\text {kin }}=65 \mathrm{~K}$. Tables 3 and 4 summarize results from $\mathrm{HCN}$ observations using HHSMT. An example of observed and modeled spectra on May 12 is given in Figure 2. Our procedure is to use water production rates, $Q_{\mathrm{H}_{2} \mathrm{O}}$, obtained by direct IR observations of water hot bands. In the case of $73 \mathrm{P}-\mathrm{B}$, we note that water production estimations at IR wavelengths vary largely, so a constant value of $1.9 \times 10^{28} \mathrm{~s}^{-1}$ is assumed (from Kobayashi et al. 2007; Bonev et al. 2008a); on May 9, the $Q_{\mathrm{H}_{2} \mathrm{O}}$ was reported to be $3.5 \times 10^{28} \mathrm{~s}^{-1}$. In the same way, we employ a mean $Q_{\mathrm{H}_{2} \mathrm{O}}$ of $1.14 \times 10^{28} \mathrm{~s}^{-1}$ for fragment C (Dello Russo et al. 2007).

Our observation coverage allows us to infer on the outgassing evolution of fragments $\mathrm{B}$ and $\mathrm{C}$ at millimeter wavelengths. As observed in Figure 3, a strong decrease of gas production of

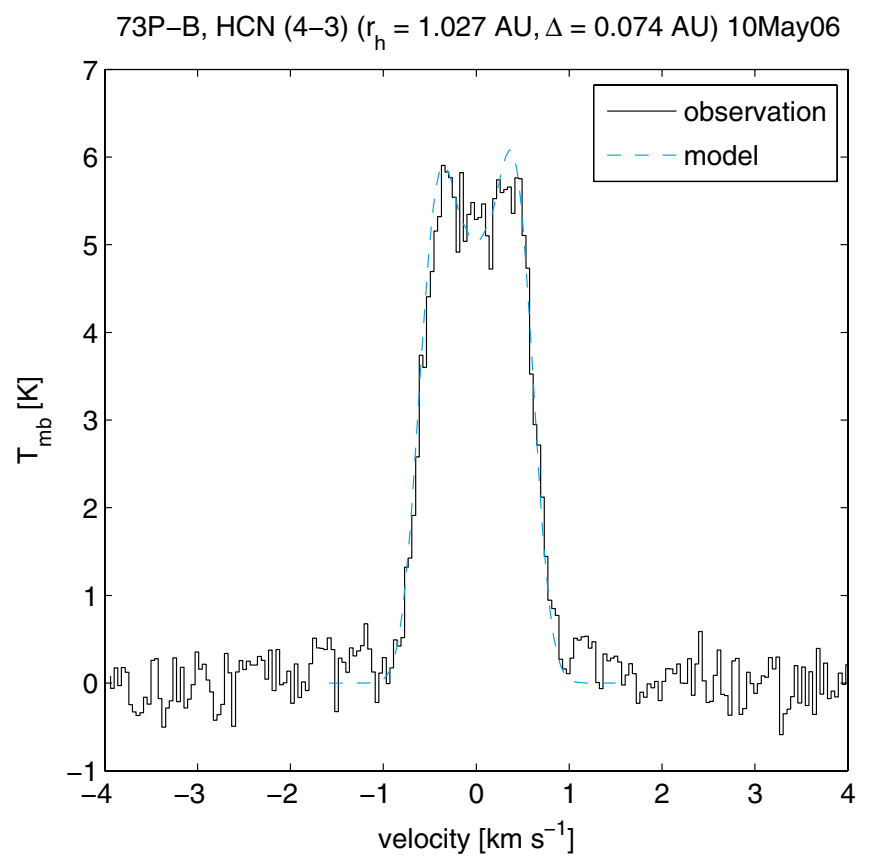

Figure 2. HCN (4-3) emission line of 73P/Schwassmann-Wachmann 3 fragment B on 2006 May 10. Black: observed spectrum, blue dashed: model result.

(A color version of this figure is available in the online journal.)

73P-B is confirmed from our observations on May 10. Existing calculations of $\mathrm{HCN}$ production rates in fragment $\mathrm{B}$ on May 

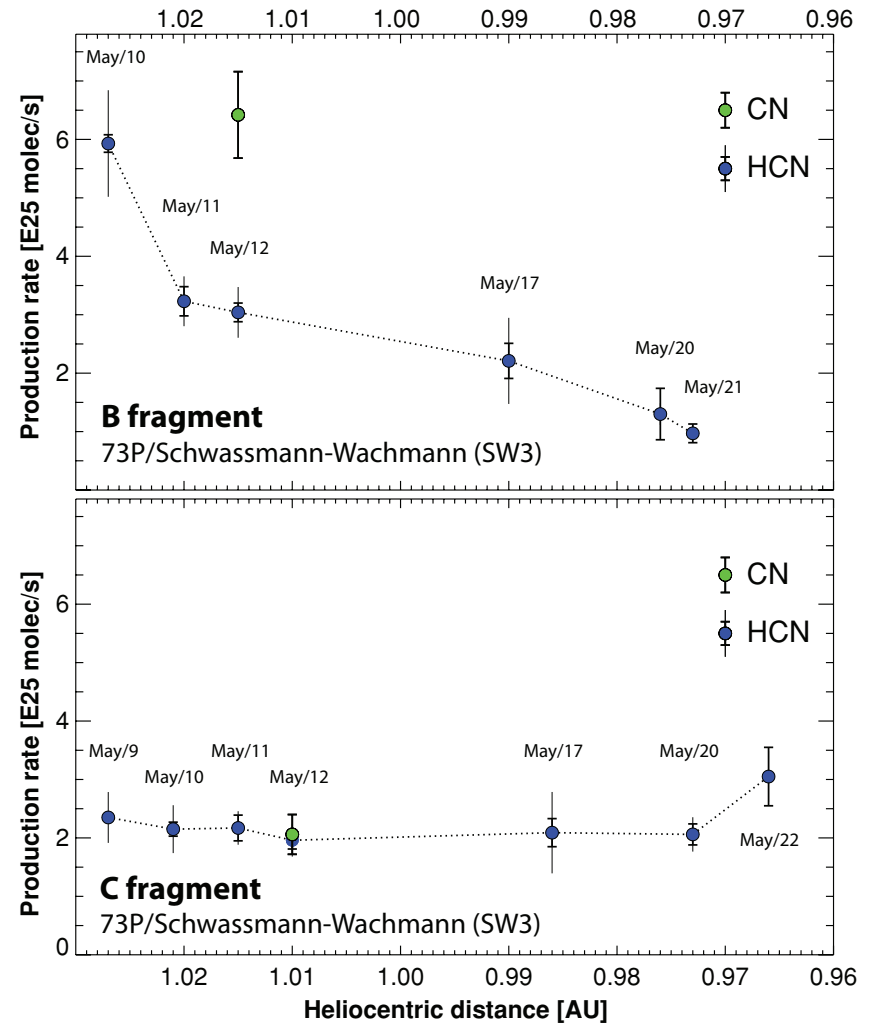

Figure 3. Production rate evolution for fragments $\mathrm{B}$ and $\mathrm{C}$ during the observational campaign. Values retrieved from the submillimeter data for $\mathrm{HCN}$ are presented with blue dots, and values for $\mathrm{CN}$ (retrieved from the optical) are presented with green dots. Stochastic uncertainties (associated with the intrinsic signal-to-noise ratio of the data) are drawn with horizontal error bars, while the systematic uncertainties are described with vertical lines. Systematic errors are introduced by transient weather conditions and were quantified by frequently measuring flux standards, and retrieving the maximum excursions in the observed fluxes.

(A color version of this figure is available in the online journal.)

10-11 (Lis et al. 2008) are similar to our estimations. It is worth noticing a decrease of 73P-B's gaseous productivity by a factor of 2 in about a week as shown by our observations. These results evidence the rapid decrease in gas productivity after the outburst event which was already noticed by observations at other wavelengths (e.g., Dello Russo et al. 2007; Bertini et al. 2009). Indeed, during the period of May 10-17, fragment B evidenced larger production rates in comparison to 73P-C, the latter showing a rather stable gas production in its trajectory toward the Sun. Subsequent observations of 73P-B after May $17\left(r_{h}=0.990 \mathrm{AU}\right)$ resulted in a lower gas productivity than that of fragment $\mathrm{C}$.

In particular, Figure 4 shows the resulting spectrum from observations of 73P-B on May 9. Its strong asymmetry might be the result of an outgassing jet expanding in a direction away from the observer (Earth). Clearly, a three-dimensional (3D) model is eventually required to model such an event.

\subsection{CN Production Rates}

To obtain the pure gas emission profiles from the images acquired with the $\mathrm{CN}$ cometary filter, it is necessary to subtract the contribution of the underlying continuum, and the way to get the continuum contribution in the gas filter can be found in detail in Lin et al. (2007). The $\mathrm{CN}$ acquired images have $27.88 \%$ and $27.55 \%$ contributions of continuum in the blue range for B and $\mathrm{C}$ fragments, respectively. Therefore, the $\mathrm{CN}$ comet images

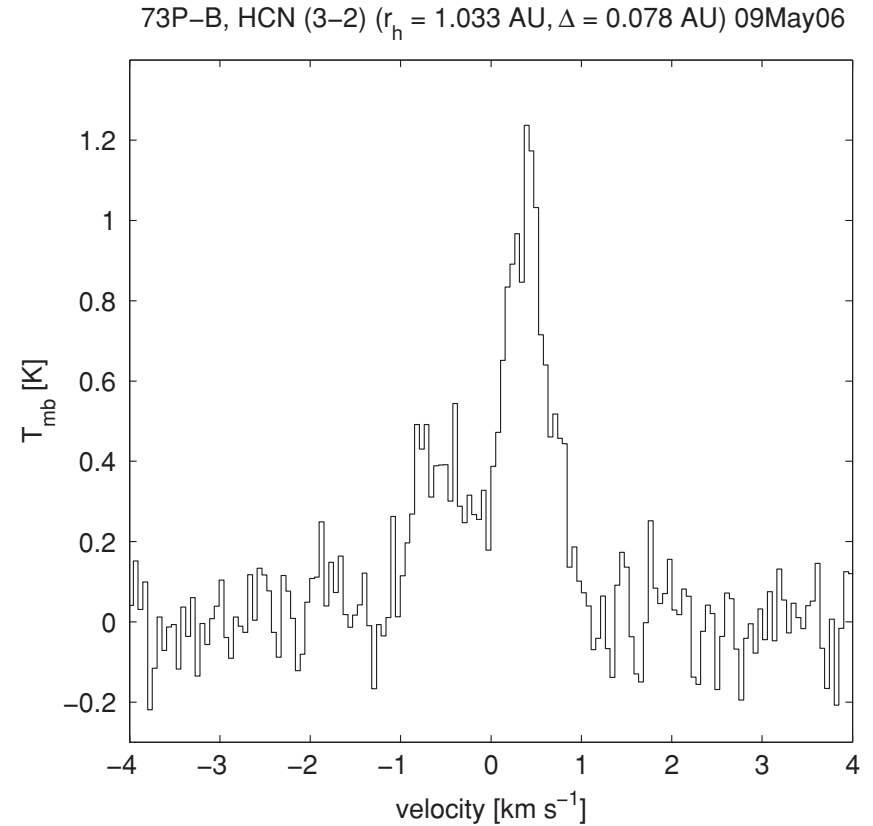

Figure 4. $\mathrm{HCN}(3-2)$ emission line of 73P/Schwassmann-Wachmann 3 fragment B obtained at the HHSMT on 2006 May 9.

result from the subtraction of the flux-calibrated images acquired with the $\mathrm{CN}$ cometary filters and the $\mathrm{BC}$ continuum images multiplied by the above-mentioned values $\left(\mathrm{CN} \approx \mathrm{CN}_{\mathrm{obs}}-0.28\right.$ $\left.\mathrm{BC}_{\mathrm{obs}}\right)$. In order to determine gas production rates by means of the Haser model (Haser 1957), we have obtained a radial emission profile of $\mathrm{CN}$ from the images at a position angle (P.A.) where no clear structure is found, and where the projected cometocentric distance is maximum (i.e., southern hemisphere). Unfortunately, the position of the optocenter, usually centered at the comet nucleus, was located in the upper left corner of the CCD frame. This prevented us from obtaining full radial profiles by azimuthal average over $360^{\circ}$, as these profiles would only reach a projected cometocentric distance of $\sim 4000 \mathrm{~km}$. Such a distance is rather meaningless when applying the Haser model, and thus this clearly represents an undesired limitation in the optical data. On the one hand, we have a good spatial resolution but, on the other hand, a short spatial coverage given by a very short geocentric distance and small CCD.

In order to derive the $\mathrm{CN}$ production rate for both fragments, we have compared the observed density radial profile of $\mathrm{CN}$ with the theoretical curve of the Haser model, assuming $v_{p}=$ $0.53 \mathrm{~km} \mathrm{~s}^{-1}$ and $0.63 \mathrm{~km} \mathrm{~s}^{-1}$ for fragments $B$ and C, respectively (obtained from our millimeter observations), customary values for the daughter velocity $v_{d}$ set to $1 \mathrm{~km} \mathrm{~s}^{-1}$, and parent and daughter scale lengths $l_{p}=13,000 \mathrm{~km}$ and $l_{d}=210,000 \mathrm{~km}$ (from A'Hearn et al. 1995), respectively. Fluorescence efficiency factors for $\mathrm{CN}$ have been computed at the corresponding heliocentric distance and velocity from tabulated values in Schleicher (1983). We have considered a radial profile at P.A.s of 206.4 and 200.8 counted from north (up in the frames) toward east (left in the frames) for B and C fragments, respectively. This allows us to obtain radial profiles of $\mathrm{CN}$ column densities reaching a (maximum) projected cometocentric distance, $\rho_{\max }$, of 11,700 for fragment $B$ and $14,000 \mathrm{~km}$ for fragment C. Figure 5 shows the observed $\mathrm{CN}$ profile for $\mathrm{B}$ and $\mathrm{C}$ fragments together with the best fit by means of the Haser model. This best fit is obtained with $Q_{\mathrm{CN}}=(6.42 \pm 0.74) \times 10^{25} \mathrm{~s}^{-1}$ for fragment B and $(2.06 \pm 0.34) \times 10^{25} \mathrm{~s}^{-1}$ for fragment $\mathrm{C}$. 


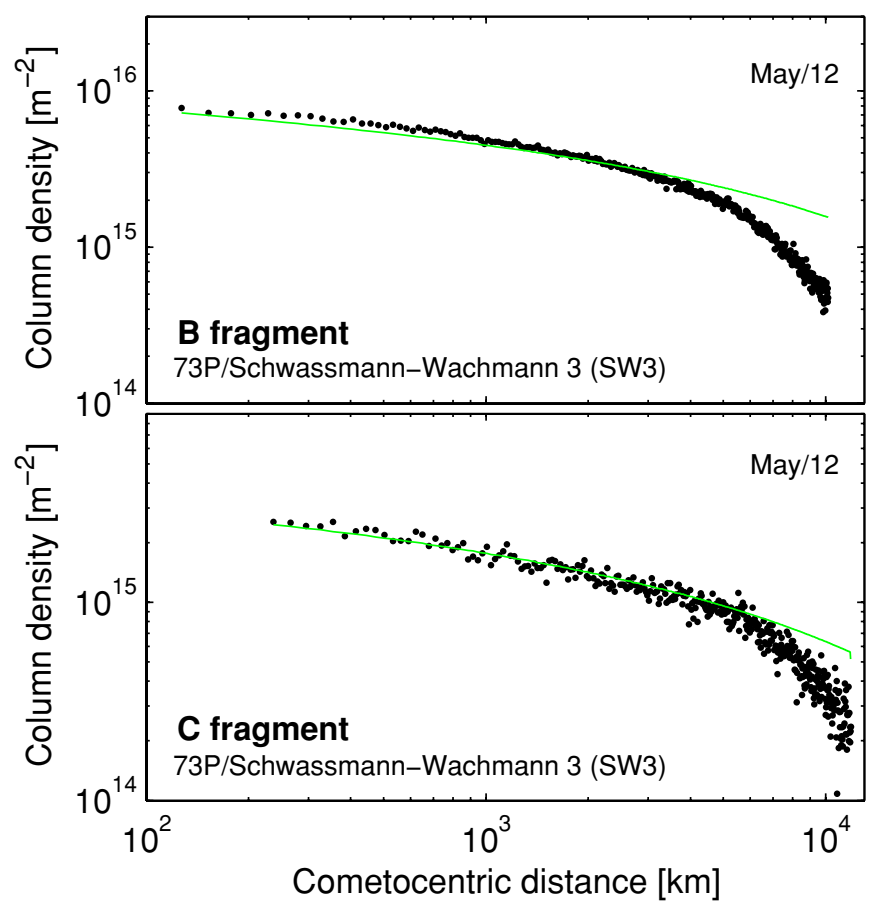

Figure 5. Observed $\mathrm{CN}$ profile for fragments B and C (black), along with the best fit by means of the Haser model (green).

(A color version of this figure is available in the online journal.)

\subsection{Mapping of HCN: Comparison to Optical Images}

Mapping of rotational emission lines has been only attempted in few comets (e.g., see Wright et al. 1998; de Pater et al. 1998; Blake et al. 1999; Lovell et al. 1999; Wink et al. 1999; Biver et al. 1999; Veal et al. 2000; Boissier et al. 2007). Position-switched mapping (PSM), which consists in moving the telescope beam across the source at a constant velocity, provides extended coverage of the molecular emissions with single-dish telescopes. On 2006 May 12, there was a unique opportunity to map the $\mathrm{HCN}$ molecule distribution in fragments $\mathrm{B}$ and C. Such spectral distribution maps were constrained by observing fragments $\mathrm{B}$ and $\mathrm{C}$ at different right ascension (R.A.) and declination (decl.) offsets from their central position. In particular, these observations were carried out during the comet's closest approach to Earth, which coincided with the period of outburst activity in fragment B (see Figure 6). As a result, additional information could be obtained on the spatial distribution by observing the line shapes projected along the line of sight (although with a limited spatial resolution of $\sim 10^{\prime \prime}$ at most).

For these observations, the orbital parameters for 73P-B were $r_{h}=1.015 \mathrm{AU}, \Delta=0.068 \mathrm{AU}$, the comet's elongation angle $(\phi$; Sun-Earth-comet $)=92^{\circ}$, comet's phase angle $(\alpha$; Sun-comet-Earth $)=84^{\circ}$, the solar P.A. of the extended radius vector $=245^{\circ}$, and the minus velocity vector $(\operatorname{PsAMV})=317^{\circ}$. Accordingly, orbital parameters for 73P-C were $r_{h}=1.010 \mathrm{AU}$, $\Delta=0.079 \mathrm{AU}, \phi=88^{\circ}, \alpha=88^{\circ}, \mathrm{P} . \mathrm{A} .=251^{\circ}$, and PsAMV $=$

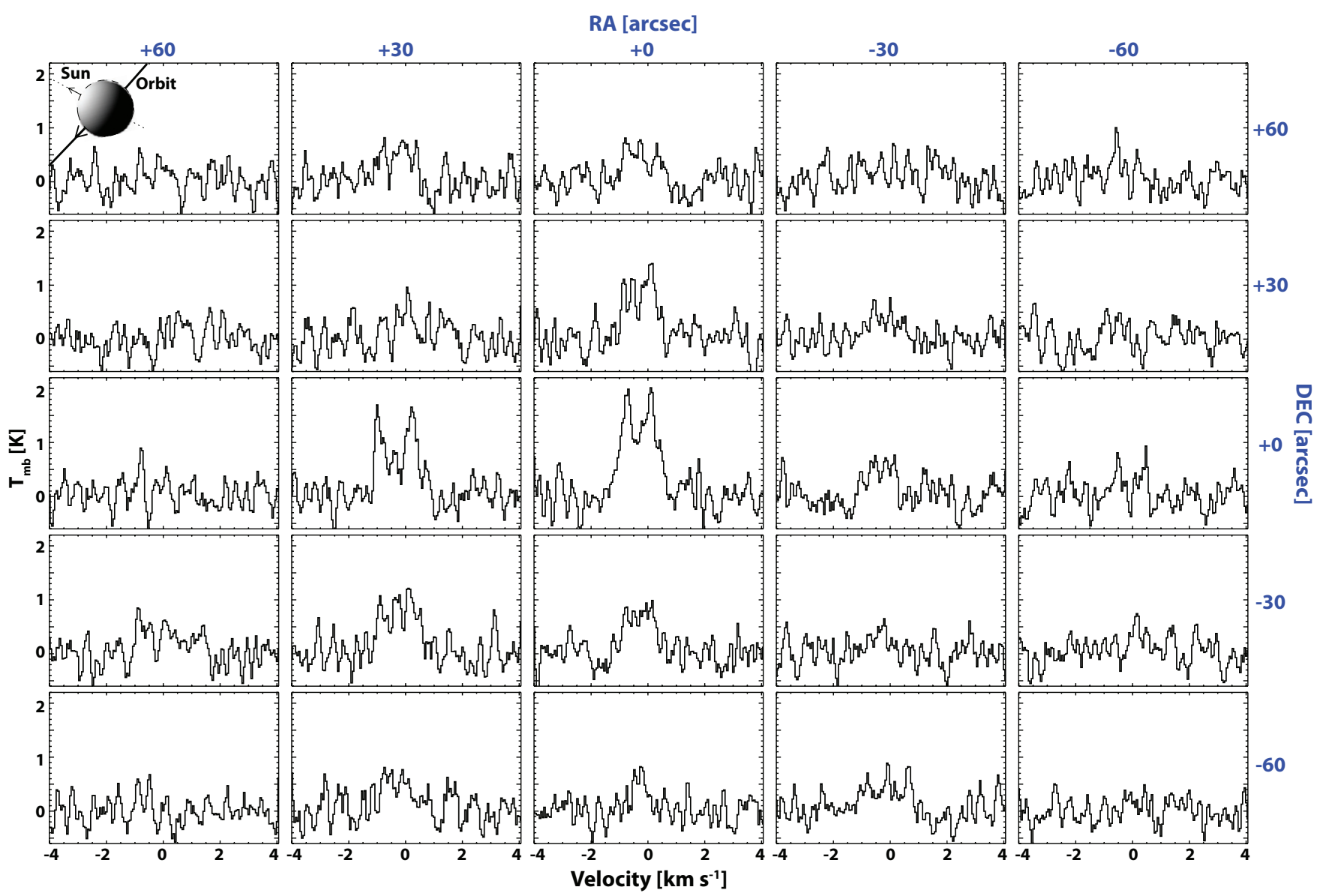

Figure 6. Spectral map of the HCN(3-2) emission line of comet 73P-B/Schwassmann-Wachmann 3 on 2006 May 12, using the PSM technique.

(A color version of this figure is available in the online journal.) 
$322^{\circ}$. Fragment B was observed between 12:25 and 14:50 UT and fragment $C$ between 9:17 and 11:40 UT.

Despite the fact that PSM involves shorter integration times and thus less signal-to-noise ratios ( $\mathrm{S} / \mathrm{Ns}$ ), fragment $\mathrm{B}$ displayed strong emission lines on May 12 which permitted maps with an FOV of $120^{\prime \prime} \times 120^{\prime \prime}$. An interesting outcome from this map (see Figure 7 (top)) is that fragment B evidenced significant ejection in the SE direction and, to a less degree, toward the NW orientation-a distribution somewhat unusual since outburst events have been usually reported to appear in solar and antisolar directions. This means that a main outburst was clearly evolving in the direction of motion of the $\mathrm{B}$ fragment during this day. Taking into account the lack of spatial resolution of millimeter mapping, this morphology agrees with arc structures reported by optical observers on previous days (Vincent et al. 2010).

Evidencing less gas production on the same day, fragment $\mathrm{C}$ was mapped with an FOV equal to $60^{\prime \prime} \times 60^{\prime \prime}$ (see Figure 8 (top)). Its millimeter map suggests outgassing features in a threeaxis configuration, i.e., toward the Sun position and a two-fold SE-NW elongation.

Comparison to optical images (bottom plots in Figures 7 and 8), with the same FOV and using the median algorithm to erase star traces, shows similar structures as those observed for $\mathrm{HCN}$ in millimeter maps. Moreover, we have used the adaptive Laplace filtering and the radial renormalization techniques to enhance any structure deviating from spherical symmetry in the gas and dust coma. As observed in Figures 9 and 10, the adaptive Laplace filtering also brings out the previously mentioned structures of CN. Similarly, the radial renormalization technique confirms the features found by Laplace filtering. We observe a low correlation between $\mathrm{CN}$ outgassing and the continuum images, i.e., the dust tail which heads toward the anti-solar direction (see bottom plots in Figures 9 and 10).

\section{DISCUSSION ON HCN-CN PARENTAGE}

The closest approach of 73P/Schwassmann-Wachmann 3 to Earth (before perihelion) gave us a unique opportunity to sample its inner coma and, thanks to the high spectral sensitivity of our submillimeter observations and a good spatial resolution of optical data, the chance to analyze particular outgassing features and characteristics close to the nucleus. In this section, we comment on jet morphologies and production rates from our joint observation on 2006 May 12, and further explore the possible role of $\mathrm{HCN}$ and dust as parents for $\mathrm{CN}$.

Having a branching ratio of $97 \%$, photodissociation of $\mathrm{HCN}$ leads to direct formation of $\mathrm{H}$ and $\mathrm{CN}$ (Huebner et al. 1992). As a result, hydrogen cyanide has been claimed as the major parent species for cometary cyanide. This affirmation, however, is a subject of extensive debate. The controversy started after determination of $\mathrm{HCN} / \mathrm{CN}$ abundances in comet IRAS-Araki-Alcock (1983d), when Bockelée-Morvan et al. (1984) measured a lower HCN production rate than that corresponding to CN. This motivated Bockelée-Morvan et al. (1985) to propose additional parent sources for cometary $\mathrm{CN}$, such as $\mathrm{HC}_{3} \mathrm{~N}, \mathrm{CH}_{3} \mathrm{CN}$, and $\mathrm{C}_{2} \mathrm{~N}_{2}$. Formation via $\mathrm{HC}_{3} \mathrm{~N}$ or $\mathrm{CH}_{3} \mathrm{CN}$ photodissociation has been shown to be minor since their production rates in C/1995 O1 (Hale-Bopp) were determined to be a factor of 10 lower than that of HCN (Bockelée-Morvan et al. 2000). The case of $\mathrm{C}_{2} \mathrm{~N}_{2}$, however, remains still inconclusive since its detection in comets has not been possible from current ground-based telescopes (due to the lack of sensitivity to detect its weak vibrational lines at infrared wavelengths).
According to Woodney et al. (2002), there exist three conditions to confirm $\mathrm{HCN}$ as the sole source for $\mathrm{CN}$, these are as follows: (1) consistency in the production rate of $\mathrm{HCN}$ and $\mathrm{CN}$, (2) agreement between the HCN photodissociation scale length and the observed CN parent scale length, and (3) a similar morphology in their radial distribution (although simultaneous investigation of all three conditions seems difficult to be accomplished.). For instance, Woodney et al. (2002) found a better correlation between $\mathrm{HCN}$ and $\mathrm{CN}$ than between $\mathrm{HCN}$ and the optically dominant dust in comet Hale-Bopp. Likewise, using the Berkeley-Illinois-Maryland Association (BIMA) array, Friedel et al. (2005) determined a consistent HCN production rate relative to that of $\mathrm{CN}$ in comet LINEAR $(\mathrm{C} / 2002$ T7). However, a similar analysis in comet NEAT (C/2001 Q4) resulted in lower $\mathrm{HCN}$ production rates, which suggested a different parent species for cyanide. Fray et al. (2005) derived an average value of $\mathrm{CN}$ parent scale lengths, considering minimum solar activity at a heliocentric distance of $1 \mathrm{AU}$, and compared $\mathrm{HCN}$ and $\mathrm{CN}$ production rates in several comets. From a total of eight comets, they found a correlation between $\mathrm{HCN}$ and $\mathrm{CN}$ production rates in one-half of the total samples only. Therefore, they concluded that an additional process is needed to explain the $\mathrm{CN}$ density observed in the remaining ones.

A particular case is that of comet $8 \mathrm{P} /$ Tuttle where, according to measurements using NIRSPEC at the Keck Observatory, Bonev et al. (2008b) claimed that HCN does not appear to be the native precursor for $\mathrm{CN}$. Such a result has been indicated also by Waniak et al. (2009), who suggested a dust source as a potential formation mechanism for $\mathrm{CN}$ species. Origin of $\mathrm{CN}$ from dust particles has already been observed by Newburn \& Spinrad (1989) and A'Hearn et al. (1995); however, the large variation among the sampled comets has denoted that formation from grains may not occur in all comets.

Investigation of $\mathrm{HCN}$ and $\mathrm{CN}$ isotopic ratios in comet C/1995 O1 (Hale-Bopp) has suggested a different source for $\mathrm{CN}$ (Manfroid et al. 2005). However, a revised analysis of Hale-Bopp's data plus recent determinations in comet 17P/ Holmes have resulted in similar $\mathrm{HCN}$ and $\mathrm{CN}$ isotopic ratios (Bockelée-Morvan et al. 2008). Thus, Bockelée-Morvan et al. (2008) concluded that $\mathrm{HCN}$ is the main parent for $\mathrm{CN}$ in cometary comae.

Synchronous mapping at multiple wavelengths is a potential tool which is starting to take importance. Even though mapping at millimeter wavelengths has already been pursued by several astronomical observers, its synergy to address the $\mathrm{HCN}-\mathrm{CN}$ parentage by means of comparison to other wavelengths has not yet been fully explored. On a previous attempt, Woodney et al. (2002) observed HCN jets in comet Hale-Bopp and combined them with near simultaneous $\mathrm{CN}$ images. The potential of this technique led them to observe similarities on production rates and radial distributions of $\mathrm{HCN}$ and $\mathrm{CN}$, but discrepancies between scale lengths. As a result, the correlation between $\mathrm{HCN}$ and $\mathrm{CN}$ was certainly not perfect.

After comparing millimeter maps and optical images, we evidence a high reciprocity on jet morphologies of $\mathrm{HCN}$ and $\mathrm{CN}$ in fragment $\mathrm{B}$ (see Figure 7). Likewise, fragment $\mathrm{C}$ denotes a similar outcome (Figure 8). Their correlation is, however, not optimally attained forbidding us to establish any definite conclusion. In addition, we clearly observe the dust tail heading to the anti-solar direction in both fragments, which suggests a low correlation between $\mathrm{CN}$ outgassing and a grain source (see Figures 9 and 10). Thereby, dust does not seem to be an effective 

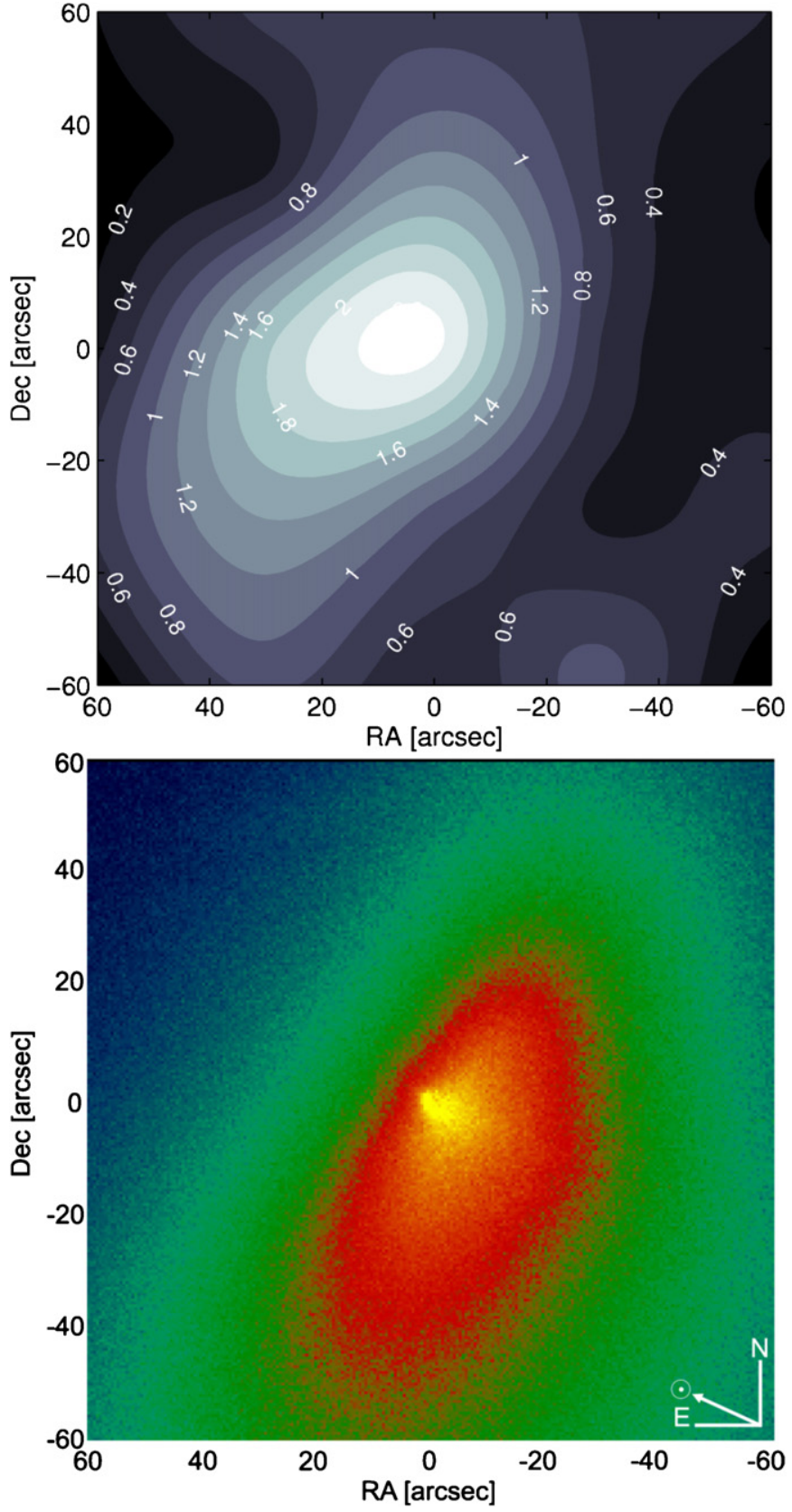

Figure 7. Comparison between $\mathrm{HCN}$ and $\mathrm{CN}$ features in fragment $\mathrm{B}$ on 2006 May 12. Top: spectral map of the $\mathrm{HCN}(3-2)$ emission line using the $10 \mathrm{~m}$ telescope at HHSMT (contours represent line-integrated intensities $\left(\mathrm{K} \mathrm{km} \mathrm{s}^{-1}\right)$ ). Bottom: optical images using the $1 \mathrm{~m}$ telescope at the Lulin Observatory (optical observations were performed with the ESA Rosetta comet filters for CN at 387/ $6 \mathrm{~nm}$ ). The FOV is $120^{\prime \prime} \times 120^{\prime \prime}$, corresponding to $\sim 6000 \times 6000 \mathrm{~km}$.

(A color version of this figure is available in the online journal.)

source of $\mathrm{CN}$ in comet 73P, though a minor contribution should not be disregarded.

These millimeter maps are treated as snapshots of the cometary coma, but the time required to complete a map was in the order of $2.3 \mathrm{hr}$. The strong rotational variability claimed by Drahus et al. (2010) would suggest undesired effects in the brightness distribution when comparing distant points in the coma, i.e., artificial coma structures induced by nucleus rotation. On the other hand, a similar distribution of the coma morphology for $\mathrm{HCN}$ and $\mathrm{CN}$ in each fragment (Figures 7 and 8) would be an argument in favor of a real spatial variation as opposed to a dominating temporal/rotation effect.
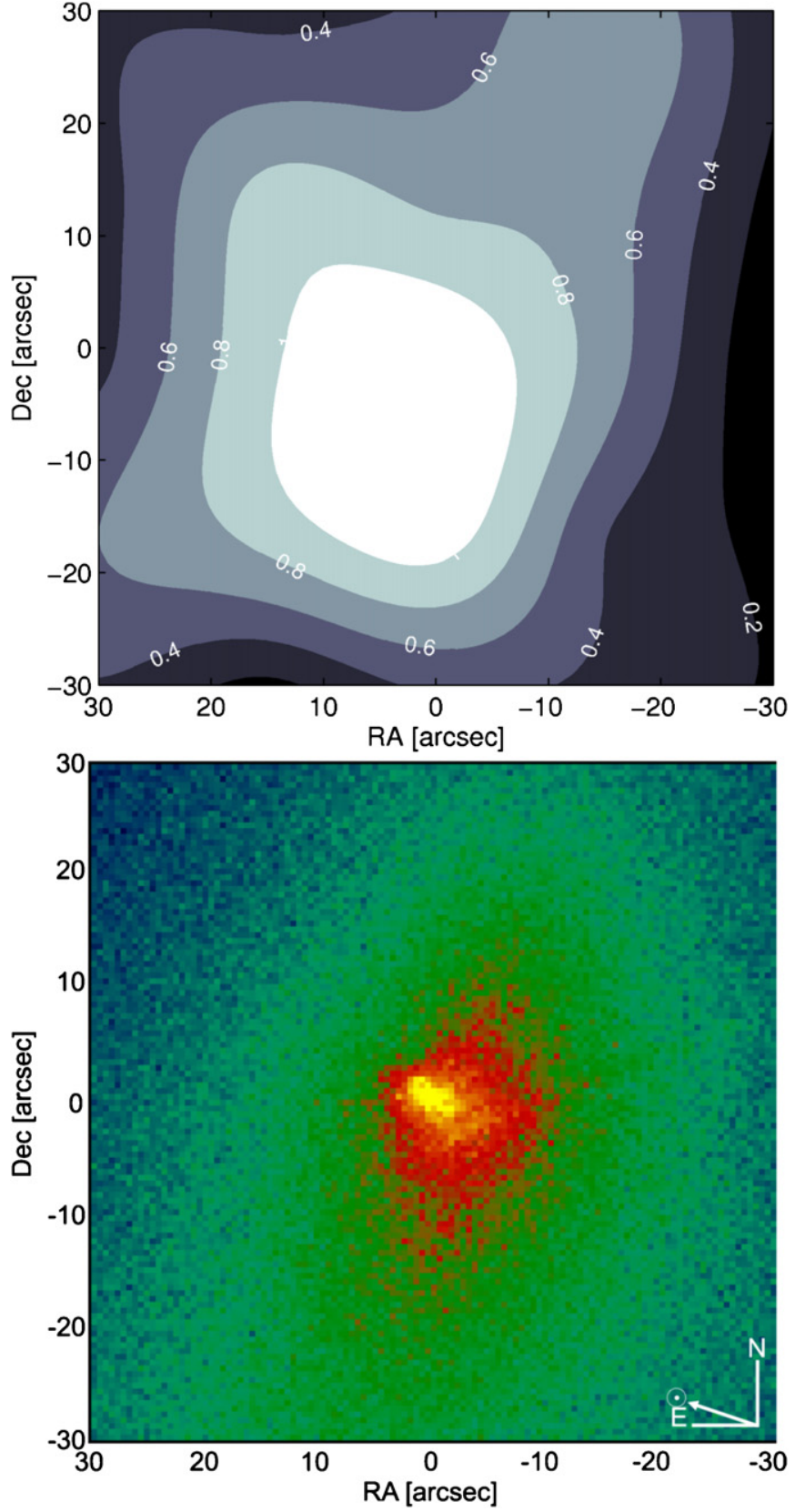

Figure 8. Comparison between $\mathrm{HCN}$ and $\mathrm{CN}$ features in fragment $\mathrm{C}$ on 2006 May 12. Top: spectral map of the $\mathrm{HCN}(3-2)$ emission line using the $10 \mathrm{~m}$ telescope at HHSMT (contours represent line-integrated intensities $\left(\mathrm{K} \mathrm{km} \mathrm{s}^{-1}\right)$ ). Bottom: optical images using the $1 \mathrm{~m}$ telescope at the Lulin Observatory. The FOV is $60^{\prime \prime} \times 60^{\prime \prime}$, corresponding to $\sim 3500 \times 3500 \mathrm{~km}$.

(A color version of this figure is available in the online journal.)

Concerning production rates, we have obtained $Q_{\mathrm{HCN}}$ equal to $(3.04 \pm 0.16) \times 10^{25} \mathrm{~s}^{-1}$ (Section 4.1) and $Q_{\mathrm{CN}}$ resulted in $(6.42 \pm 0.74) \times 10^{25} \mathrm{~s}^{-1}$ (see Section 4.2) for fragment B. In the case of fragment C, $Q_{\mathrm{HCN}}$ equals $(1.83 \pm 0.13) \times 10^{25} \mathrm{~s}^{-1}$ and $Q_{\mathrm{CN}}$ is $(2.06 \pm 0.34) \times 10^{25} \mathrm{~s}^{-1}$. Thus, comparison of $\mathrm{HCN}$ and $\mathrm{CN}$ production rates in fragment $\mathrm{C}$ indicates a mean value of $Q_{\mathrm{CN}}$ slightly larger than $Q_{\mathrm{HCN}}$, if we bear in mind the estimated error bars and standard uncertainties these rates confirm hydrogen cyanide as the main source for the $\mathrm{CN}$ radical. In the case of fragment $\mathrm{B}$, it is evident that $\mathrm{CN}$ production exceeds that of $\mathrm{HCN}$ by a factor of 2 . However, it is important to mention that direct comparison of $\mathrm{HCN}$ and $\mathrm{CN}$ production 

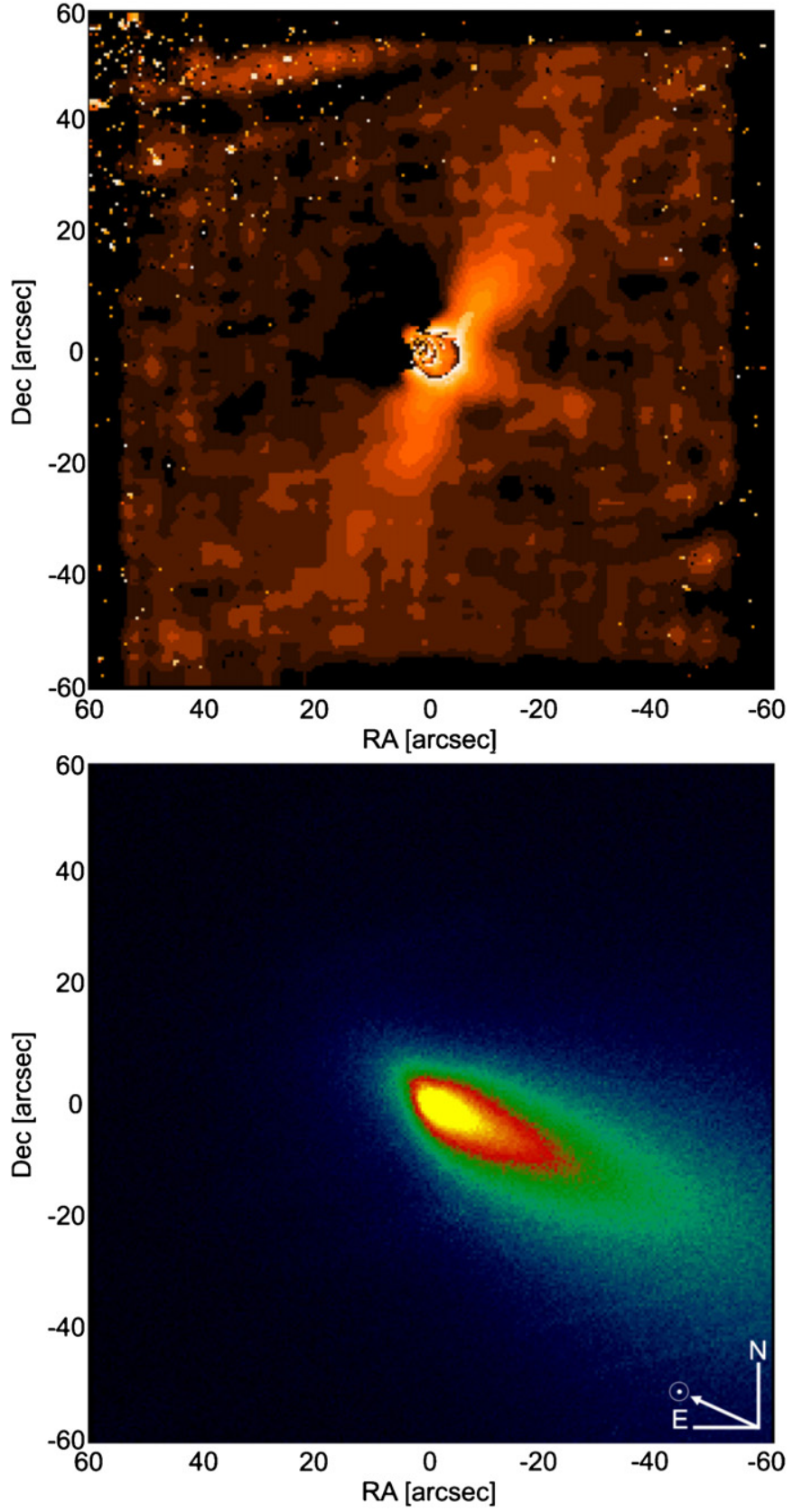

Figure 9. Comparison between $\mathrm{CN}$ structures and dust in fragment B. Top: adaptive Laplace filtering method of the $\mathrm{CN}$ image in Figure 7 (bottom). Bottom optical images using the ESA Rosetta comet filters for the blue continuum at $445 / 4 \mathrm{~nm}$.

(A color version of this figure is available in the online journal.)

rates is ill-conditioned. In order to derive production rates, we have assumed spherically symmetric outflow and steady production over the lifetime of the parent volatile. Similar to the impact event on comet Tempel 1, the rapid decrease in spectral intensity produced by outburst activity in fragment B demonstrates that steady-state production was not achieved, and thus these production rates should be interpreted as indicators of activity (Mumma et al. 2005). Moreover, the daughter scale length of $210,000 \mathrm{~km}$ corresponds to a timescale of $\sim 2.4$ days for the $\mathrm{CN} \rightarrow \mathrm{C}+\mathrm{N}$ dissociation, i.e., $\mathrm{CN}$ measures the production rate at a slightly different time than $\mathrm{HCN}$, and much of the $\mathrm{CN}$ observed on May 12 may still be from the $\mathrm{HCN}$ outburst on previous days. A time-dependent model (as opposed
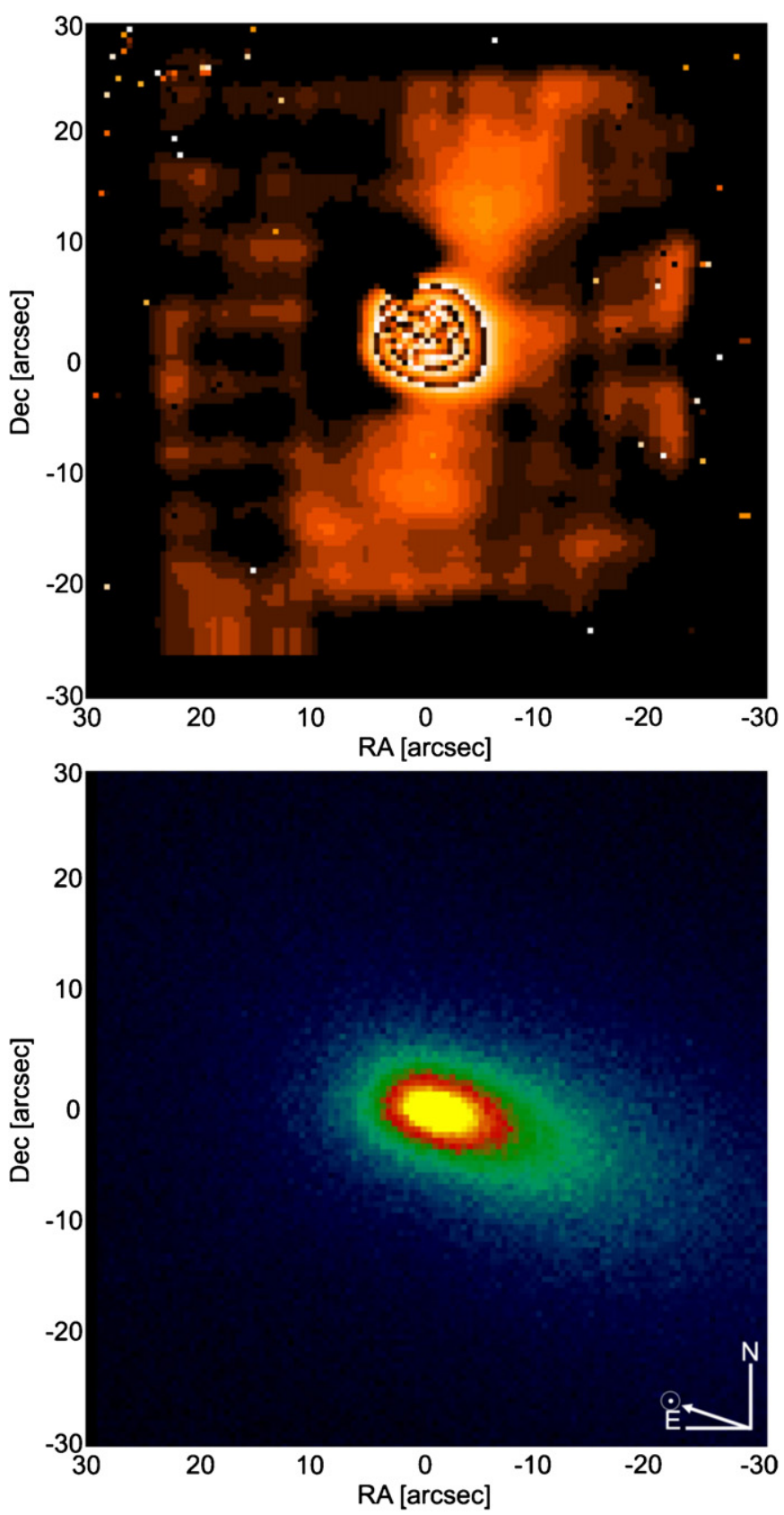

Figure 10. Comparison between $\mathrm{CN}$ structures and dust in fragment $\mathrm{C}$. Top: adaptive Laplace filtering method of the $\mathrm{CN}$ image in Figure 8 (bottom). Bottom: optical images using the ESA Rosetta comet filters for the blue continuum at $445 / 4 \mathrm{~nm}$.

(A color version of this figure is available in the online journal.)

to a steady-state model) is needed to evaluate if the HCN and $\mathrm{CN}$ production rates for fragment $\mathrm{B}$ are consistent with $\mathrm{HCN}$ as the sole source or not. This model is beyond the scope of this paper, but clearly a proper interpretation of the $\mathrm{HCN}-\mathrm{CN}$ link in the case of impulsive events (e.g., in fragment B) requires such a model before suggesting alternative sources for cyanide.

Similar to Woodney et al. (2002), we also observe large variation in rates for $\mathrm{CN}$ production in fragment B. Among others, these undesired effects are mostly introduced by uncertainties in model parameters, such as $\mathrm{CN}$ lifetimes and scale lengths, which are not accurately known. As a result, a conclusion suggesting similar production rates for $\mathrm{HCN}$ and $\mathrm{CN}$ is certainly constrained by the model parameters assumed in our calcu- 
lations. Upcoming studies by other observers would provide complementary information to establish any further conclusion. Indeed, we note a lack of published $\mathrm{CN} / \mathrm{H}_{2} \mathrm{O}$ abundances on 2006 May 12. For instance, a report of $\mathrm{CN} / \mathrm{H}_{2} \mathrm{O}$ abundances at optical wavelengths resulted in $0.28 \%$ and $0.20 \%$ for fragments $\mathrm{B}$ and $\mathrm{C}$ on May 14, respectively (Kanda et al. 2008). These estimations are in accordance with $\mathrm{HCN} / \mathrm{H}_{2} \mathrm{O}$ abundances at IR wavelengths on the same day (Dello Russo et al. 2007).

In addition to an equivalent outgassing production and a similar morphological structure of the observed species, a full analysis of $\mathrm{HCN}-\mathrm{CN}$ parentage requires the determination (and agreement) of the parent photodissociation scale length and the daughter formation scale length (as was mentioned above). In our case, the intrinsic limitations of our observations make difficult the retrieval of reliable scale lengths for comet 73P, ultimately preventing us to rule out alternative mechanisms for cyanide production.

Last but not least, it is important to remark the large fluctuation encountered in the chemical taxonomy among different comets (even in the same nucleus as observed by Deep Impact; Mumma et al. 2005; Feaga et al. 2007); this suggests that comets underwent complex thermochemical processes at different regions within the protoplanetary disk (Morbidelli et al. 2008). As it was mentioned, several investigations about the relation of hydrogen cyanide with $\mathrm{CN}$ have guided astronomical observers to a wide variety of conclusions. In particular, some studies have established hasty conclusions based on a particular observed comet. The large chemical diversity encountered in cometary composition, however, clearly indicates that more evidence is required to put some light into this debate, most probably on a statistical basis.

\section{CONCLUSIONS}

Parent volatiles released by the nucleus were analyzed by means of their rotational transitions at millimeter wavelengths. These allowed us to provide $\mathrm{HCN}$ production rates for fragments $\mathrm{B}$ and $\mathrm{C}$, and thus their gas evolution, at heliocentric distances between 0.966 and 1.033 AU before perihelion. Furthermore, our observations provided unprecedented coverage shortly after the observed increase in outgassing activity of fragment B, estimated to have happened on 2006 May 8-9. During these days, outgassing ejecta enhanced gas productivity in $73 \mathrm{P}-\mathrm{B}$ surpassing that of fragment $\mathrm{C}-\mathrm{a}$ behavior which was not observed previously during 73P's apparition in 2006 and that finished $\sim 8$ days later according to our estimations.

In addition, we have discussed the role of $\mathrm{HCN}$ and dust as parent sources for $\mathrm{CN}$, and the potential synergy between observations at different wavelengths. This study suggests a high correlation between $\mathrm{HCN}$ and $\mathrm{CN}$, rather than an origin from a grain source, and thus supports previous investigations indicating $\mathrm{HCN}$ as a major source for $\mathrm{CN}$ (provided that we only consider analysis of jet morphologies and production rates). Nevertheless, the presence of an alternative source for cyanide could not be fully ruled out. Clearly, the larger sensitivity and spatial resolution attained by upcoming interferometers, such as ALMA, will certainly enhance previous studies and provide some light into this discussion.

This work is based on joint observations from the Heinrich Hertz Submillimeter Telescope and Lulin Observatory. We are grateful to the staff of HHSMT and LO for their great assistance and generous allocation of telescope time throughout our observational campaign. L.P. acknowledges D. BockeléeMorvan, N. Biver, K. Jockers, M. Drahus, M. Lippi, and M. J. Mumma for useful discussions, the anonymous referee for valuable comments on the manuscript, and the support of this research by the Max-Planck-Gesellschaft. L.M.L. and Z.-Y.L. thank the support given by the Spanish Ministerio de Educación y Ciencia and Ministerio de Ciencia e Innovación under projects ESP2006-02934 and AyA 2009-08011. The SMT is operated by the Arizona Radio Observatory (ARO), Steward Observatory, University of Arizona.

\section{REFERENCES}

A'Hearn, M. F., Millis, R. L., Schleicher, D. G., Osip, D. J., \& Birch, P. V. 1995, Icarus, 118, 223

Baars, J. W. M., Martin, R. N., Mangum, J. G., McMullin, J. P., \& Peters, W. L. 1999, PASP, 111, 627

Bensch, F., \& Bergin, E. A. 2004, ApJ, 615, 531

Bertini, I., Lara, L. M., Vincent, J.-B., Boehnhardt, H., Küppers, M., \& Rodrigo, R. 2009, A\&A, 496, 235

Biver, N. 1997, PhD thesis, Univ. Paris VII

Biver, N., et al. 1999, AJ, 118, 1850

Blake, G. A., Qi, C., Hogerheijde, M. R., Gurwell, M. A., \& Muhleman, D. O. 1999, Nature, 298, 213

Bockelée-Morvan, D., Crovisier, J., Mumma, M. J., \& Weaver, H. A. 2004, in Comets II, ed. M. C. Festou, H. U. Keller, \& H. A. Weaver (Tucson, AZ: Univ. Arizona Press), 391

Bockelée-Morvan, D., et al. 1984, A\&A, 141, 411

Bockelée-Morvan, D., et al. 1985, A\&A, 151, 90

Bockelée-Morvan, D., et al. 2000, A\&A, 353, 1101

Bockelée-Morvan, D., et al. 2008, ApJ, 679, L49

Böhnhardt, H., Kaufl, H. U., Keen, R., Camilleri, P., Carvajal, J., \& Hale, A 1995, IAU Circ., 6274, 1

Boissier, J., Bockelée-Morvan, D., Biver, N., Crovisier, J., Despois, D., Marsden, B. G., \& Moreno, R. 2007, A\&A, 475, 1131

Bonev, B. P., Mumma, M. J., Kawakita, H., Kobayashi, H., \& Villanueva, G. L. 2008a, Icarus, 196, 241

Bonev, B. P., Mumma, M. J., Radeva, Y. L., DiSanti, M. A., Gibb, E. L., \& Villanueva, G. L. 2008b, ApJ, 680, L61

Combi, M. R., et al. 1999, ApJ, 512, 961

Crovisier, J. 1984, A\&A, 130, 361

Crovisier, J., \& Encrenaz, Th. 1983, A\&A, 126, 170

Dello Russo, N., Vervack, R. J., Weaver, H. A., Biver, N., Bockelée-Morvan, D., Crovisier, J., \& Lisse, C. M. 2007, Nature, 448, 172

de Pater, I., et al. 1998, AJ, 116, 987

Drahus, M., Küppers, M., Jarchow, C., Paganini, L., Hartogh, P., \& Villanueva, G. L. 2010, A\&A, 510, 55

Faure, A., et al. 2007, MNRAS, 382, 840

Feaga, L. M., et al. 2007, Icarus, 190, 284

Fray, N., Bénilan, Y., Cottin, H., Gazeau, M.-C., \& Crovisier, J. 2005, Planet. Space Sci., 53, 1243

Friedel, D. N., et al. 2005, ApJ, 630, 623

Fuse, T., et al. 2007, PASJ, 59, 381

Haser, L. 1957, Bull. Soc. R. Sci. Liege, 43, 740

Hogerheijde, M. R., \& van der Tak, F. F. S. 2000, A\&A, 362, 697

Huebner, W. F. Keady, J. J., \& Lyon, S. P. 1992, Ap\&SS, 195, 1

Jones, T. J., et al. 2008, AJ, 135, 1318

Kanda, Y.-i., Mori, A., Kobayashi, H., \& Kawakita, H. 2008, PASJ, 60, 1191

Kinoshita, D., et al. 2005, Chin. J. Astron. Astrophys., 5, 315

Kobayashi, H., Kawakita, H., Mumma, M. J., Bonev, B. P., Watanabe, J., \& Fuse, T. 2007, ApJ, 668, L75

Lin, Z. Y., et al. 2007, A\&A, 469, 771

Lin, Z. Y., et al. 2009, AJ, 138, 625

Lis, D. C., et al. 2008, ApJ, 675, 931

Lovell, A. J., et al. 1999, Earth Moon Planets, 77, 253

Manfroid, J., et al. 2005, A\&A, 432, L5

Morbidelli, A., Levison, H. F., \& Gomes, R. 2008, in The Solar System Beyond Neptune, ed. M. A. Barucci, H. Boehnhardt, D. P. Cruikshank, \& A. Morbidelli (Tucson, AZ: Univ. Arizona Press), 275

Mumma, M. J., et al. 2005, Science, 310, 270

Newburn, R. L., \& Spinrad, H. 1989, AJ, 97, 552

Pickett, H. M., Poynter, R. L., Cohen, E. A., Delitsky, M. L., Pearson, J. C., \& Muller, H. S. P. 1998, J. Quant. Spectrosc. Radiat. Transfer, 60, 883

Reach, W. T., et al. 2009, Icarus, 203, 571 
Rothman, L. S., et al. 2009, J. Quant. Spectrosc. Radiat. Transfer, 110, 533

Sault, R. J., Teuben, P. J., \& Wright, M. C. H. 1995, in ASP Conf. Ser. 77 , Astronomical Data Analysis Software and Systems IV, ed. R. A. Shaw,

H. E. Payne, \& J. J. E. Hayes (San Francisco, CA: ASP), 433

Schleicher, D. G. 1983, PhD thesis, Univ. Maryland

Ulich, B. L., \& Haas, R. W. 1976, ApJS, 30, 247

Veal, J. M., et al. 2000, AJ, 119, 1498

Villanueva, G. L., et al. 2006, ApJ, 650, L87
Vincent, J.-B., Böhnhardt, H., Bertini, I., Lara, L.-M., Küppers, M., \& Rodrigo, R. 2010, Earth Moon Planets, 106, 27

Waniak, W., Borisov, G., Drahus, M., Bonev, T., Czart, K., \& Küppers, M. 2009, Earth Moon Planets, 105, 327

Weaver, H. A., \& Mumma, M. J. 1984, ApJ, 276, 782

Wink, J., et al. 1999, Earth Moon Planets, 78, 63

Woodney, L. M., et al. 2002, Icarus, 157, 193

Wright, M. C. H., et al. 1998, AJ, 116, 3018

Xie, X., \& Mumma, M. J. 1992, ApJ, 386, 720 